\title{
Children of Treatment-Seeking Depressed Mothers: A Comparison With the Sequenced Treatment Alternatives to Relieve Depression (STAR ${ }^{\star}$ D) Child Study
}

Lisa A. Batten, M.A., Mariely Hernandez, M.A., Daniel J. Pilowsky, M.D., M.P.H., Jonathan W. Stewart, M.D., Pierre Blier, M.D., Ph.D., Martine F. Flament, M.D., Ph.D., Ernest Poh, M.S., Priya Wickramaratne, Ph.D., Myrna M. Weissman, Ph.D.

\begin{abstract}
Objective: To estimate the prevalence of current psychiatric disorders among children and adolescents (collectively called children) of mothers entering treatment for depression; to examine maternal predictors of child psychopathology among children of depressed mothers; and to determine consistency of findings with a similar child study ancillary to Sequenced Treatment Alternatives to Reduce Depression $\left(\mathrm{STAR}^{\star} \mathrm{D}\right)$ from seven United States sites (STAR ${ }^{\star} \mathrm{D}$-Child). Method: Mothers $(\mathrm{N}=82)$ with major depressive disorder (MDD) enrolled in a treatment study in Ottawa (Ontario, Canada) or New York City, and their eligible children $(\mathrm{N}=145)$ (aged 7 through 17 years) were assessed independently when the mother enrolled. Results: Among the children of depressed mothers, $42 \%$ had at least one current psychiatric diagnosis, including affective $(15 \%)$, anxiety $(19 \%)$, behavioral $(23 \%)$, and/or substance use (2\%) disorders. In all, $40 \%$ of the children were rated as impaired by clinical assessors. Mothers' comorbid anxiety disorders predicted the highest rates of current disorders in the child in both studies. The severity of the mother's depression predicted behavioral problems in the child. The current and lifetime rates of psychiatric disorders in the children of depressed mothers were compared to rates found in STAR ${ }^{\star} \mathrm{D}$ Child and findings were consistent. Both studies used similar diagnostic assessments. Conclusion: Given the high prevalence of offspring psychiatric disorders, inquiring about the mental health of the children when a depressed mother comes for treatment, and referring children for treatment when appropriate, are important. J. Am. Acad. Child Adolesc. Psychiatry; 2012; 51(11):1185-1196.

Key Words: depressed mothers, offspring, treatment seeking, STAR ${ }^{\star} \mathrm{D}-\mathrm{Child}$, high risk.
\end{abstract}

$\mathrm{N}$ umerous studies have shown that the children of depressed as compared with nondepressed parents have increased lifetime rates of depressive, anxiety, and behavioral disorders. ${ }^{1,2}$ These children are also at a greater risk for social, cognitive, and medical difficulties that continue into adulthood, extending risks to the next generation. ${ }^{1,2}$ The type of disorder in children varies by their age. ${ }^{3}$ The mechanism of transmission is unclear, ${ }^{1}$ but the findings are consistent across studies regardless of site or design. These findings in children are primarily with depressed mothers, reflecting the lower rate of depression in men and their tendency not to seek treatment. ${ }^{4}$

Recent studies suggest that the successful treatment of depressed mothers to remission is associated with reduction of symptoms in the children. ${ }^{1,5-7}$ These results were enduring over the subsequent years after maternal remission. ${ }^{8}$ The results were not consistent across all groups, with children of single and/or anxious mothers having the lowest rate of maternal remission and poorest child outcomes even if the mother remitted. ${ }^{9}$ 
Children with symptoms at the time the mother enters treatment for depression could provide an opportunity for intervention for mothers and their children. However, studies of offspring at high risk due to parents' depression focus on lifetime illness rather than co-occurring psychiatric illnesses. Studies demonstrating increased lifetime risk in children of depressed parents are less useful for understanding opportunities for immediate intervention. Previously, in a study ancillary to Sequenced Treatment Alternatives to Reduce Depression $\left(\mathrm{STAR}^{\star} \mathrm{D}\right)$ based in seven sites in the United States, we showed that children whose mothers were seeking treatment for major depressive disorder (MDD) were at high risk for a current psychiatric illness themselves. ${ }^{10,11}$ We report findings from a new study carried out at New York City and Ottawa (Ontario, Canada). Neither site was involved in the STAR ${ }^{\star} \mathrm{D}$ Child study. We report baseline current prevalence rates of psychiatric disorders in children whose depressed mothers were enrolled in a doubleblind treatment study for MDD. The purpose is to determine the sturdiness of the original STAR ${ }^{*} \mathrm{D}$ Child findings, and to determine whether similar maternal characteristics predicted child illness.

\section{METHOD}

\section{Sample}

The sample consisted of 93 parents who entered a clinical trial, hereafter the "Adult Study," and their 168 children or adolescents (collectively referred to as children) aged 7 through 17 years. Parents were considered eligible for the Child Study if they participated in the Adult Study and had at least one eligible child aged 7 through 17 years who lived or spent at least half of the time with the treated parent, and did not have a developmental disability that would preclude them from participating in diagnostic interviews. All willing eligible parents and children were enrolled. Although the Child Study did not provide child treatment, children were not excluded from the study if they were in treatment. The Child Study baseline assessment was completed within 2 weeks of the parents' randomization in the Adult Study. The protocol for the Child Study was reviewed and approved by the institutional review boards at each site. Written informed consent from parents and children was obtained.

Adult Study participants were outpatients aged 18 through 65 years, with nonpsychotic MDD, and without a lifetime diagnosis of bipolar disorder, schizophrenia, schizoaffective disorder, or a current substance use disorder. Patients with current medical and psychiatric conditions, except as noted above, were included unless a medical condition contraindicated the use of one of the randomly assigned study medications. Adult Study participants received a battery of assessments by the Adult Study team. The child assessments were conducted by independent assessors who were aware of the parent's depression but did not have access to parental depression assessments. Adult Study participants were randomized to one of three treatment conditions, i.e., bupropion, escitalopram, or both medications, using a double-blind design.

\section{Assessments}

The Adult Study assessments are described below.

The Schedule for Clinical Interview for DSM-IV Diagnoses, Axis I, Patient Version (SCID-IV-I/P) is a semistructured interview for making DSM-IV diagnoses with acceptable reliability for most disorders, including MDD and depressive subtypes, melancholia, and atypical depression. ${ }^{12}$

The Montgomery-Asberg Depression Rating Scale (MADRS) is a measure of overall severity of depressive symptoms. Ten items, each rated on a scale of 0 to 6 , are included. ${ }^{13}$ Scores below 10 are indicative of remission or absence of clinically significant depression. A MADRS score of 22 or higher was required for a parent's entry into the Adult Study.

The 17-item Hamilton Rating Scale for Depression (HAMD-17) assesses the severity of depressive symptoms. ${ }^{14}$ Each symptom has three to five possible responses that increase in severity, and total scores range from 0 to 54 . Adult Study participants were classified as either moderately (HAMD-17 score of 1421) or markedly (HAMD-17 score of $\geq 22$ ) depressed at baseline.

The Child Study assessments are described in the following section.

All children were directly assessed by experienced interviewers using the Kiddie Schedule for Affective Disorders and Schizophrenia-Present and Lifetime Version (KSADS-PL) (described below), separately and before the mother's interview. During the child interview, a rating of child symptoms was made, and, similarly, the mother's report of the child's symptoms was rated during the mother's interview. The rater made a final judgment about each symptom (summary scores) at the end of both interviews, and this was reviewed by a clinician where there was disagreement. The child assessors were not part of the Adult Study team and did not rate the mothers' clinical state.

The Kiddie-Schedule for Affective Disorders and Schizophrenia-Present and Lifetime Version (K-SADS-PL), a diagnostic instrument to assess most Axis I DSM-IV disorders in children and adolescents. ${ }^{15}$ Children and parents were interviewed separately (see above), and summary scores for each symptom were used to make $D S M-I V$ diagnoses. Diagnoses, as well as counts of 
child-reported and parent-reported symptoms included in the screening section of the K-SADS, were used to prepare this report. To relieve participant burden, only disorders known to be highly prevalent among children of depressed parents were included. Eighteen DSM-IV disorders of children were included (affective, anxiety, disruptive behavior, and substance use disorders).

The Child Global Assessment Scale (C-GAS), a clinician-rated estimate of overall functioning and severity of disturbance, was completed using all available information about the child. ${ }^{16}$ Scores range between 0 and 100, with scores above 90 indicating superior functioning, and scores below 70 indicating impaired global functioning.

\section{Interviewer Training and Quality Assurance}

Six interviewers with prior clinical and/or research experience with children and adolescents underwent training in the study assessments under the supervision of an experienced child psychiatrist (D.J.P.). After the initial 2-day training, each interviewer observed three live interviews before conducting a supervised interview. After completion of training, at least one directly observed interview and an acceptable reliability with the supervisor were required. Reliabilities between the interviewers and either D.J.P. or M.F.F. during the course of the study were obtained by comparing interviewers' ratings of a child from audiotape, scored by D.J.P. or M.F.F. The mean $\kappa$ value for agreement on symptoms was 0.64 (range, 0.54-0.81) and for diagnoses it was 0.92 (range, 0.60-1.00). Quality assurance activities included review of children's diagnostic assessments, monthly conference calls, and site visits to ascertain compliance and review of record to ensure completion and check for logical inconsistencies. All assessment procedures were similar to those used in the STAR *D Child Study. ${ }^{7}$

\section{Data Analysis}

Preliminary analysis of group differences and the mean values for continuous outcomes were tested using the $t$ test, and categorical outcomes were compared by the $\chi^{2}$ test or Fisher's exact test, depending on the number of observations in a cell. Subsequent analyses of child diagnoses adjusting for the potential confounding effects of age and gender of child and nonindependence of outcomes for offspring from the same family were conducted as follows: When the analysis focused on the association between child diagnoses and specific variables (i.e., site), child diagnosis was considered as the dependent variable, and the specific variable of interest was considered to be the predictor variable in a logistic regression analysis. Age and gender of child were considered a priori to be confounding variables and were included as independent variables in the analysis. When the focus was on estimating the effect of child gender on the prevalence rates of child diagnoses, adjusted odds ratios were obtained from logistic regressions with child gender as the predictor variable adjusting for the potential confounding effects of age of child. All logistic regression analyses were performed within the framework of the generalized estimating equations (GEE) approach ${ }^{17}$ by means of the GENMOD procedure within the SAS software package, to obtain estimates adjusted for potential nonindependence of outcomes of offspring from the same family. ${ }^{18}$

\section{RESULTS}

Participating parents and children were recruited at both sites between October 2007 and August 2011. Of 319 subjects, 110 parents had age-eligible children; of these 93 (82 mothers and 11 fathers; $85 \%$ ) agreed to participate in the Child Study. The 93 parents had 175 age-eligible children, of whom $168(96 \%)$ participated in the Child Study (145 children of participating mothers and 23 children of participating fathers). Of the seven nonparticipating children, three were found not to reside with the index parent at least $50 \%$ of the time, and four children declined to participate. Because of the small sample of fathers and our interest in comparing results to STAR*D-Child which included only mothers, we have not included fathers in subsequent analyses.

\section{Characteristics of Site and Mothers}

In all, 82 mothers and their 145 children participated in the study. Mother age (mean, 40.8 years and 41.0 years in Ottawa and New York, respectively), mean number of children, severity of depression, and chronicity did not differ by site (Table 1). There were substantial educational, ethnic, marital status, employment, income, and some clinical differences between sites, reflecting the divergent sociodemographics between sites. Compared to Ottawa, New York parents were more likely to be melancholic and to have comorbid anxiety disorders. There were no statistically significant site differences by children's age, gender, or educational level. However, children in New York were significantly less likely to live with both biological parents.

\section{Children's Diagnoses}

Table 2 shows children's current and lifetime psychiatric diagnoses at baseline by site. At baseline, $42 \%$ of the children were diagnosed with a current psychiatric disorder. The rates 
TABLE 1 Sociodemographic Characteristics of Mothers and Children, by Site

\begin{tabular}{|c|c|c|c|}
\hline Mothers & $\begin{array}{l}\text { OHtawa } \\
(n=41)\end{array}$ & $\begin{array}{c}\text { New York } \\
(\mathrm{n}=41)\end{array}$ & Statistic $^{a}$ \\
\hline Age, mean (SD) & $40.8(5.7)$ & $41 \quad(7.0)$ & -0.12 \\
\hline \multicolumn{4}{|l|}{ Education, $\mathrm{n}(\%)$} \\
\hline Some high school or less & $2(4.9)$ & $15(36.6)$ & \multirow[t]{4}{*}{$15.15^{\star \star}$} \\
\hline High school graduate & $9(22.0)$ & $10(24.4)$ & \\
\hline Some college & $12(29.3)$ & 7 (17.1) & \\
\hline College graduate + & $18(43.9)$ & $8(19.5)$ & \\
\hline \multicolumn{4}{|l|}{ Ethnicity, n (\%) } \\
\hline White & 37 (90.2) & $1 \quad(2.4)$ & \multirow[t]{4}{*}{$69.54^{\star \star}$} \\
\hline Black & $1(2.4)$ & $1 \quad(2.4)$ & \\
\hline Hispanic & $1 \quad(2.4)$ & $38(92.7)$ & \\
\hline Other $^{b}$ & $2(4.9)$ & $1(2.4)$ & \\
\hline \multicolumn{4}{|l|}{ Marital status, n (\%) } \\
\hline Married with spouse & $23(56.1)$ & $11(26.8)$ & \multirow[t]{2}{*}{$6.8^{\star \star}$} \\
\hline Single/divorced/separated & $18(43.9)$ & 29 (70.7) & \\
\hline \multicolumn{4}{|l|}{ Employment status, n (\%) } \\
\hline Employed full-time/part-time & $30(73.2)$ & $15(36.6)$ & \multirow{4}{*}{$18.66^{* *}$} \\
\hline Homemaker & $1 \quad(2.4)$ & $3(7.3)$ & \\
\hline Full-time student/unemployed & $4 \quad(9.8)$ & $20(48.8)$ & \\
\hline Other & $6(14.6)$ & $2(4.9)$ & \\
\hline Receiving public assistance, $\mathrm{n}(\%)$ & $5(12.2)$ & $25(61.0)$ & $22.98^{* *}$ \\
\hline \multicolumn{4}{|l|}{ Household income, $n(\%)$} \\
\hline Less than $\$ 15 \mathrm{~K}$ & $3 \quad(7.3)$ & $26(63.4)$ & \multirow{3}{*}{$42.28^{* \star}$} \\
\hline$\$ 15-39 \mathrm{~K}$ & $4 \quad(9.8)$ & $8(19.5)$ & \\
\hline More than $\$ 40 \mathrm{~K}$ & $33(80.5)$ & $4 \quad(9.8)$ & \\
\hline Children per family, mean (SD) & $1.9(0.9)$ & $1.7(1.1)$ & 0.8 \\
\hline \multicolumn{4}{|l|}{ Clinical characteristics } \\
\hline HAMD-17, mean (SD) & $21.9(4.3)$ & $22(4.4)$ & -0.1 \\
\hline Chronic MDE, n $(\%)^{c}$ & $19(46.3)$ & $18(43.9)$ & 0.01 \\
\hline \multicolumn{4}{|l|}{ Type of depression } \\
\hline Atypical & $10(24.4)$ & $7(17.1)$ & 0.67 \\
\hline Melancholic & $12(29.3)$ & $4 \quad(9.8)$ & $4.97^{*}$ \\
\hline \multicolumn{4}{|l|}{ MDD severity (current), $n(\%)^{d}$} \\
\hline Mild & $2(4.9)$ & $2(4.9)$ & \multirow[b]{4}{*}{$15.42^{* *}$} \\
\hline Moderate & $14(34.1)$ & $13(31.7)$ & \\
\hline Severe & $25 \quad(61)$ & $25(61)$ & \\
\hline Any anxiety disorder, n (\%) & $6(14.6)$ & $23(56.1)$ & \\
\hline Children & $(n=76)$ & $(n=69)$ & Statistica $^{a}$ \\
\hline Age, mean $(S D)$ & $11.5(3.4)$ & $12.4(3.3)$ & -1.55 \\
\hline \multicolumn{4}{|l|}{ Living status, n (\%) } \\
\hline Two-parent householde & 55 (72.4) & $23(33.3)$ & \multirow[t]{2}{*}{$22.17^{\star *}$} \\
\hline Mother only & $21(27.6)$ & $46(66.7)$ & \\
\hline Female, n (\%) & $36(47.4)$ & $34(49.3)$ & 0.05 \\
\hline \multicolumn{4}{|l|}{ Education, n (\%) } \\
\hline Grade 1-6 & $38(50.0)$ & $29(42.0)$ & \multirow[t]{2}{*}{0.92} \\
\hline Above 6th Grade & $38(50.0)$ & $40(58.0)$ & \\
\hline \multicolumn{4}{|c|}{$\begin{array}{l}\text { Note: Numbers vary because of missing data. MDD }=\text { major depressive disorder. } \\
\text { at Statistic for age comparisons, and } \chi^{2} \text { statistic or Fisher's exact test for all other comparisons. } \\
\text { bIncludes one American Indian, one parent who identified as more than one race, and one unknown. } \\
\text { "Duration of current major depressive episode (MDE) } \geq 2 \text { years. } \\
\text { dSeverity defined as mild: Hamilton Rating Score for Depression } 17 \text {-them (HAMD-17) }<14 \text {, moderate: } 14 \leq \text { HAMD-17 } \leq 21 \text {, severe: HAMD-17 } \geq 22 \\
\text { eIncludes any combination of biological, adopted, or stepmother and stepfather. } \\
{ }^{*} \mathrm{p}<.05 ;{ }^{*} p<.01 \text {. }\end{array}$} \\
\hline
\end{tabular}


TABLE 2 Current and Lifetime Prevalence and Odds of Children's Psychiatric Disorders by Site, Among Children of Depressed Mothers

\begin{tabular}{|c|c|c|c|c|c|c|c|c|}
\hline & \multicolumn{4}{|c|}{ Current Diagnosis, n (\%) } & \multicolumn{4}{|c|}{ Lifetime Diagnosis, n (\%) } \\
\hline & $\begin{array}{c}\text { All } \\
(\mathrm{N}=145)\end{array}$ & $\begin{array}{l}\text { Ottawa } \\
(n=76)\end{array}$ & $\begin{array}{c}\text { New York } \\
(n=69)\end{array}$ & OR $(95 \% \mathrm{Cl})$ & $\begin{array}{c}\text { All } \\
(\mathrm{N}=145)\end{array}$ & $\begin{array}{l}\text { Oftawa } \\
(n=76)\end{array}$ & $\begin{array}{c}\text { New York } \\
(n=69)\end{array}$ & OR $(95 \% \mathrm{Cl})$ \\
\hline Affective disorder, any & $22(15.2)$ & $9(11.8)$ & $13(18.8)$ & $0.5(0.2-1.3)$ & $31(21.4)$ & $13(17.1)$ & $18(26.1)$ & $0.8(0.4-1.8)$ \\
\hline Adjustment disorder with depressed mood & $1(0.7)$ & $1(1.3)$ & $0(0.0)$ & $-^{a}$ & $2(1.4)$ & $2(2.6)$ & $0(0.0)$ & $-^{a}$ \\
\hline Depressive disorder NOS & $6(4.1)$ & $3(3.9)$ & $3(4.3)$ & $0.8(0.2-4.3)$ & $6(4.1)$ & $3(3.9)$ & $3(4.3)$ & $1.2(0.2-6.1)$ \\
\hline Dysthymia & $2(1.4)$ & $1(1.3)$ & $1(1.4)$ & $0.8(0.1-13.9)$ & $2(1.4)$ & $1(1.3)$ & $1(1.4)$ & $1.2(0.1-19.2)$ \\
\hline Major depressive disorder & $13(9.0)$ & $4(5.3)$ & $9(13.0)$ & $0.3(0.1-1.2)$ & $21(14.5)$ & $7(9.2)$ & $14(20.3)$ & $0.5(0.2-1.4)$ \\
\hline Anxiety disorder, any & $28(19.3)$ & $11(14.5)$ & $17(24.6)$ & $0.5(0.2-1.1)$ & $35(24.1)$ & $15(19.7)$ & $20(29.0)$ & $0.8(0.4-1.8)$ \\
\hline Agoraphobia & $1(0.7)$ & $0(0.0)$ & $1(1.4)$ & $-^{a}$ & $1(0.7)$ & $0(0.0)$ & $1(1.4)$ & $-^{a}$ \\
\hline GAD/overanxious & $6(4.1)$ & $3(3.9)$ & $3(4.3)$ & $0.8(0.2-4.3)$ & $8(5.5)$ & $5(6.6)$ & $3(4.3)$ & $2.0(0.5-8.9)$ \\
\hline Obsessive-compulsive disorder & $2(1.4)$ & $1(1.3)$ & $1(1.4)$ & $-^{a}$ & $2(1.4)$ & $1(1.3)$ & $1(1.4)$ & $-^{a}$ \\
\hline Posttraumatic stress disorder & $1(0.7)$ & $1(1.3)$ & $0(0.0)$ & $-^{a}$ & $5(3.4)$ & $2(2.6)$ & $3(4.3)$ & $0.8(0.1-4.8)$ \\
\hline Separation anxiety & $14(9.7)$ & $4(5.3)$ & $10(14.5)$ & $0.3(0.1-1.0)$ & 15 (10.3) & $4(5.3)$ & $11(15.9)$ & $0.4(0.1-1.3)$ \\
\hline Simple phobia & $7(4.8)$ & $1(1.3)$ & $6(8.7)$ & $0.1(0.0-1.1)$ & $7(4.8)$ & $1(1.3)$ & $6(8.7)$ & $0.2(0.0-1.6)$ \\
\hline Social phobia & $5(3.4)$ & $2(2.6)$ & $3(4.3)$ & $0.6(0.1-3.4)$ & $6(4.1)$ & $3(3.9)$ & $3(4.3)$ & $1.2(0.2-6.1)$ \\
\hline Behavioral disorder, any & $34(23.4)$ & $15(19.7)$ & $19(27.5)$ & $0.6(0.3-1.3)$ & $39(26.9)$ & $16(21.1)$ & $23(33.3)$ & $0.8(0.4-1.6)$ \\
\hline ADHD (all subtypes) & $29(20.0)$ & 15 (19.7) & $14(20.3)$ & $0.9(0.4-2.0)$ & $31(21.4)$ & $15(19.7)$ & $16(23.2)$ & $1.1(0.5-2.5)$ \\
\hline Conduct disorder & $3(2.1)$ & $2(2.6)$ & $1(1.4)$ & $-^{a}$ & $6(4.1)$ & $3(3.9)$ & $3(4.3)$ & $1.2(0.2-6.1)$ \\
\hline Oppositional defiant disorder & $11(7.6)$ & $2(2.6)$ & $9(13.0)$ & $0.2(0.0-0.8)^{*}$ & 15 (10.3) & $3(3.9)$ & $12(17.4)$ & $0.3(0.1-1.0)$ \\
\hline Substance use disorder, any & $3(2.1)$ & $2(2.6)$ & $1(1.4)$ & $1.7(0.2-19.5)$ & $5(3.4)$ & $4(5.3)$ & $1(1.4)$ & $4.9(0.5-45.5)$ \\
\hline Alcohol abuse & $0(0.0)$ & $0(0.0)$ & $0(0.0)$ & $-^{a}$ & $1(0.7)$ & $1(1.3)$ & $0(0.0)$ & $-{ }^{a}$ \\
\hline Cannabis abuse & $0(0.0)$ & $0(0.0)$ & $0(0.0)$ & $-^{a}$ & $2(1.4)$ & $2(2.6)$ & $0(0.0)$ & $-^{a}$ \\
\hline Cannabis dependence & $2(1.4)$ & $2(2.6)$ & $0(0.0)$ & $-^{a}$ & $2(1.4)$ & $2(2.6)$ & $0(0.0)$ & $-^{a}$ \\
\hline Other drug dependence & $1(0.7)$ & $0(0.0)$ & $1(1.4)$ & $-^{a}$ & $1(0.7)$ & $0(0.0)$ & $1(1.4)$ & $--^{a}$ \\
\hline Any disorder & $61(42.1)$ & $27(35.5)$ & $34(49.3)$ & $0.5(0.2-1.0)$ & $68(46.9)$ & $29(38.2)$ & $39(56.5)$ & $0.8(0.4-1.5)$ \\
\hline Impaired global functioning, $n(\%)^{b}$ & $58(40.0)$ & $21(27.6)$ & $37(53.6)$ & $\chi^{2}=10.18^{\star \star}$ & & & & \\
\hline Child reporting one or more symptoms, $n(\%)^{c}$ & $73(50.3)$ & $32(42.1)$ & $41(59.4)$ & $\chi^{2}=4.34^{*}$ & & & & \\
\hline $\begin{array}{l}\text { Note: Number of distinct families in the table }=82 \text { mother } \\
\text { intrafamily correlation. ADHD = attention-deficit/hype } \\
\text { "Could not be computed because of sparse data. } \\
\text { bScore }<70 \text { based on the Child-Global Assessment } \\
\text { "Assessed using the Kiddie Schedule for Affective Diso } \\
{ }^{*} p<.05 ;{ }^{* *}<<.01 \text {. }\end{array}$ & and Schizophr & cause of missin & hildren IK-SA & |l summary ("Any") cate & ies are adjusted & child age, ger & living situation, & other's income, and \\
\hline
\end{tabular}


TABLE 3 Adjusted Odds ${ }^{a}$ and Proportion of Children With Current Psychiatric Disorders, by Current Maternal Characteristics and Selected Demographics ${ }^{b}$

\begin{tabular}{|c|c|c|c|}
\hline & \multicolumn{2}{|c|}{ Affective } & \multirow{2}{*}{$\frac{\text { Anxiety }}{n(\%)}$} \\
\hline & n (\%) & OR $(95 \% \mathrm{Cl})$ & \\
\hline \multicolumn{4}{|l|}{ Maternal characteristics } \\
\hline \multicolumn{4}{|l|}{ Type of depression } \\
\hline \multicolumn{4}{|l|}{ Atypical } \\
\hline Yes $(n=33)$ & $6(18)$ & $1.4(0.5-4.2)$ & $5(15)$ \\
\hline No $(n=112)$ & $16(14)$ & & $23(21)$ \\
\hline \multicolumn{4}{|l|}{ Melancholic } \\
\hline Yes $(n=33)$ & $8(24)$ & $2.9(1-8.3)$ & $11(33)$ \\
\hline No $(n=112)$ & $14(13)$ & & $17(15)$ \\
\hline \multicolumn{4}{|l|}{ Chronicity of current MDE } \\
\hline$<2$ Years $(\mathrm{n}=80)$ & $12(15)$ & & $17(21)$ \\
\hline$\geq 2$ Years $(n=64)$ & $10(16)$ & $0.9(0.3-2.4)$ & $10(16)$ \\
\hline \multicolumn{4}{|l|}{ MDD severity (current) ${ }^{c}$} \\
\hline Mild/moderate $(\mathrm{n}=55)$ & $6(11)$ & & 10 (18) \\
\hline Severe $(n=86)$ & $16(19)$ & $2(0.7-5.7)$ & $18(21)$ \\
\hline \multicolumn{4}{|l|}{ Comorbid conditions $^{d}$} \\
\hline \multicolumn{4}{|l|}{ Any anxiety disorder } \\
\hline Yes $(n=49)$ & $8(16)$ & $1.3(0.5-3.5)$ & $16(33)$ \\
\hline No $(n=96)$ & $14(15)$ & & 12 (13) \\
\hline \multicolumn{4}{|l|}{ Social phobia } \\
\hline Yes $(n=12)$ & $2(17)$ & $1.2(0.2-5.9)$ & $5(42)$ \\
\hline No $(n=133)$ & $20(15)$ & & 23 (17) \\
\hline \multicolumn{4}{|l|}{ PTSD } \\
\hline Yes $(n=12)$ & $2(17)$ & $1.3(0.2-7)$ & $6(50)$ \\
\hline No $(n=133)$ & $20(15)$ & & 22 (17) \\
\hline \multicolumn{4}{|c|}{ Panic disorder with Agoraphobia } \\
\hline Yes $(n=12)$ & $5(42)$ & $4.2(1.1-16.8)^{*}$ & $4(33)$ \\
\hline No $(n=133)$ & $17(13)$ & & 24 (18) \\
\hline \multicolumn{4}{|l|}{ Demographic characteristics } \\
\hline \multicolumn{4}{|l|}{ Household income } \\
\hline Less than $\$ 15 \mathrm{~K}$ (reference) & $11(24)$ & 1.0 & $9(20)$ \\
\hline$\$ 15-39 K$ & $4(15)$ & $0.8(0.2-2.7)$ & $8(30)$ \\
\hline More than $\$ 40 K$ & $5(8)$ & $0.3(0.1-0.9)^{*}$ & $9(14)$ \\
\hline \multicolumn{4}{|l|}{ Single mother } \\
\hline Yes $(n=67)$ & $11(16)$ & $1.1(0.4-2.7)$ & $11(16)$ \\
\hline No $(n=78)$ & $11(14)$ & & $17(22)$ \\
\hline \multicolumn{4}{|c|}{ 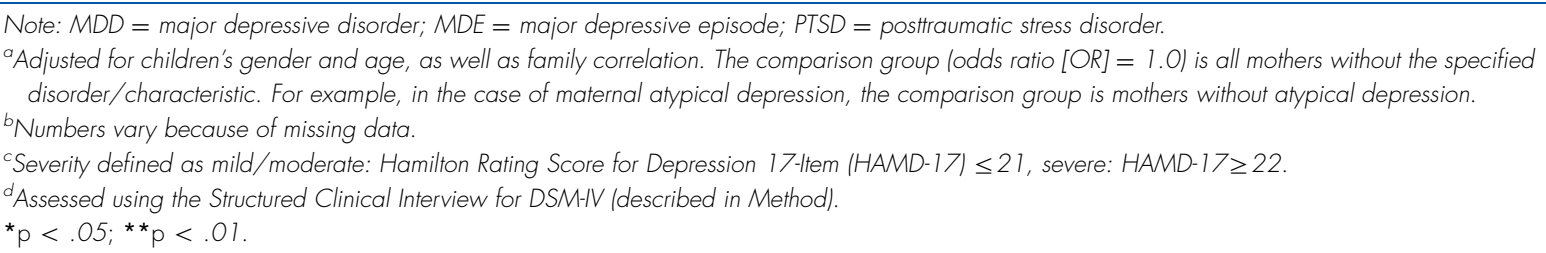 } \\
\hline
\end{tabular}

for current affective, anxiety, and behavioral disorders were $15 \%, 19 \%$, and $23 \%$ respectively. Current behavioral disorders tended to be more prevalent in boys than in girls ( $28 \%$ vs. $19 \%)$, but this difference was not statistically significant (data not shown). Of the children, $47 \%$ received a lifetime psychiatric diagnosis, including affective $(21 \%)$, anxiety $(24 \%)$, and behavioral $(27 \%)$ disorders. With the exception of current oppositional defiant disorder (13\% in New York versus $3 \%$ in Ottawa; $p<.05)$, there were no statistically significant differences in the prevalence of children's psychiatric disorders by site. The rates were mainly lower in Ottawa than in New York, although these differences did not reach statistical significance, probably because of limited 
TABLE 3 Continued

\begin{tabular}{|c|c|c|c|c|}
\hline \multirow{2}{*}{$\frac{\text { Anxiety }}{\text { OR }(95 \% \text { Cl) }}$} & \multicolumn{2}{|c|}{ Behavioral } & \multicolumn{2}{|c|}{ Any Disorder } \\
\hline & $n(\%)$ & OR $(95 \% \mathrm{Cl})$ & $n(\%)$ & OR $(95 \% \mathrm{Cl})$ \\
\hline $0.8(0.3-2.4)$ & $\begin{array}{r}6(18) \\
28(25)\end{array}$ & $0.8(0.3-2.1)$ & $\begin{array}{l}13(39) \\
48(43)\end{array}$ & $1.1(0.5-2.4)$ \\
\hline $2.6(1.1-6.3)^{*}$ & $\begin{array}{l}10(30) \\
24(21)\end{array}$ & $1.5(0.6-3.5)$ & $\begin{array}{l}18(55) \\
43(38)\end{array}$ & $1.9(0.8-4.4)$ \\
\hline $0.7(0.3-1.7)$ & $\begin{array}{l}18(23) \\
15(23)\end{array}$ & $1.1(0.5-2.4)$ & $\begin{array}{l}33(41) \\
27(42)\end{array}$ & $1(0.5-2.1)$ \\
\hline $1.2(0.5-2.8)$ & $\begin{array}{r}8(15) \\
26(30)\end{array}$ & $2.6(1.1-6.2)^{*}$ & $\begin{array}{l}20(36) \\
41(48)\end{array}$ & $1.6(0.8-3.3)$ \\
\hline $3.9(1.6-9.4)^{* *}$ & $\begin{array}{l}14(29) \\
20(21)\end{array}$ & $1.7(0.8-3.8)$ & $\begin{array}{l}27(55) \\
34(35)\end{array}$ & $2.7(1.3-5.7)^{* *}$ \\
\hline $3.2(0.9-11.5)$ & $\begin{array}{r}5(42) \\
29(22)\end{array}$ & $2.4(0.7-8.3)$ & $\begin{array}{r}6(50) \\
55(41)\end{array}$ & $1.4(0.4-4.4)$ \\
\hline $5.7(1.6-20.3)^{* *}$ & $\begin{array}{r}5(42) \\
29(22)\end{array}$ & $2.2(0.6-7.6)$ & $\begin{array}{r}8(67) \\
53(40)\end{array}$ & $2.7(0.7-9.6)$ \\
\hline $2.5(0.7-9.1)$ & $\begin{array}{r}5(42) \\
29(22)\end{array}$ & $2.5(0.7-8.8)$ & $\begin{array}{r}8(67) \\
53(40)\end{array}$ & $2.7(0.8-9.3)$ \\
\hline $\begin{array}{l}1.0 \\
1.6(0.5-4.8) \\
0.6(0.2-1.7)\end{array}$ & $\begin{array}{r}11(24) \\
8(30) \\
13(20)\end{array}$ & $\begin{array}{l}1.0 \\
1.1(0.4-3.4) \\
0.7(0.3-1.9)\end{array}$ & $\begin{array}{l}23(51) \\
13(48) \\
21(32)\end{array}$ & $\begin{array}{l}1.0 \\
0.9(0.3-2.4) \\
0.5(0.2-1.1)\end{array}$ \\
\hline $0.8(0.3-2)$ & $\begin{array}{l}19(28) \\
15(19)\end{array}$ & $1.8(0.8-4.2)$ & $\begin{array}{l}30(45) \\
31(40)\end{array}$ & $1.3(0.7-2.6)$ \\
\hline
\end{tabular}

statistical power. Lower rates in Ottawa were expected, given the higher incomes and fewer single parents. ${ }^{9}$ Lifetime behavioral disorders and attention-deficit/hyperactivity disorder (ADHD) tended to be more prevalent in boys as compared to girls (33\% versus $20 \%, p<.10)$. Of the children, $40 \%$ were impaired based on the clinician's global assessment, and 50.3\% endorsed at least one symptom on the K-SADS. These rates were higher in New York than in Ottawa.

\section{Associations With Features of Maternal Depression}

Table 3 shows the associations between current maternal clinical characteristics and current child 
TABLE 4 Multivariate Analysis of Maternal Depression Characteristics Associated With Child Psychiatric Disorders

\begin{tabular}{|c|c|c|c|}
\hline Child Psychiatric Disorder & Maternal Predictors & Adjusted OR & Statistic $^{b}$ \\
\hline \multirow[t]{2}{*}{ Child affective disorder } & Income over $\$ 40 \mathrm{~K}$ vs. under $\$ 15 \mathrm{~K}$ & $0.9(0.8-1.0)$ & 3.56 \\
\hline & Panic disorder with Agoraphobia & $1.1(0.8-1.5)$ & 0.50 \\
\hline \multirow[t]{2}{*}{ Child anxiety disorder } & Melancholic depression & $1.2(1.1-1.5)$ & $7.44^{* *}$ \\
\hline & Any anxiety disorder (maternal) & $1.3(1.1-1.5)$ & $12.46^{* *}$ \\
\hline Child behavioral disorder & Severe MDD vs. mild/moderate MDD & $1.2(1.0-1.3)$ & $5.30^{*}$ \\
\hline Child any disorder & Any anxiety disorder (maternal) & $1.2(1.0-1.5)$ & $5.04^{*}$ \\
\hline \multicolumn{4}{|c|}{$\begin{array}{l}\text { Note: } M D D=\text { major depressive disorder; } O R=\text { odds ratio. } \\
\text { aOne multivariate logistic regression model for each of the child psychiatric disorders (affective, anxiety, behavioral, anyl. The child disorder was the binary } \\
\text { dependent variable. All maternal characteristics found to be significant predictors in univariate analyses are shown, and were entered simultaneously in the } \\
\text { multivariate model. All models are adjusted for child age and gender, and for family correlation. } \\
{ }^{b} \chi^{2} \text { statistic for odds comparisons. } \\
{ }^{*} p<.05 ;{ }^{* *} p<.01 \text {. }\end{array}$} \\
\hline
\end{tabular}

disorders. Maternal melancholic depression was associated with increased odds of affective and anxiety disorders in their offspring. Children of severely depressed mothers were more than twice as likely to have behavioral disorders as compared to children of mild/moderately depressed mothers (odds ratio $[\mathrm{OR}]=2.6 ; 95 \%$ confidence interval $[\mathrm{CI}]=1.1-6.2)$.

Maternal concurrent Axis I comorbidities were also associated with a higher prevalence of current childhood disorders. Children of mothers with comorbid anxiety disorders were approximately four times more likely to have an anxiety disorder compared to children of mothers without anxiety disorders (OR $=3.9 ; 95 \% \mathrm{CI}=1.6-9.4)$. A higher maternal family income (more than $\$ 40,000)$ was associated with a decreased likelihood of offspring affective disorders $(\mathrm{OR}=0.3$; $95 \% \mathrm{CI}=0.1-0.9)$.

To determine the relative impact of severity, variables in Table 3 that were found to be significantly associated with child disorders were included simultaneously in a logistic regression model (discussed in Method) while controlling for child age, gender, and sibling correlation. Results in Table 4 show that maternal anxiety disorders significantly predicted any child diagnosis, as well as child anxiety disorders. Maternal melancholic depression predicted child anxiety disorders, and severity of maternal MDD predicted behavioral disorders.

\section{Comparisons With STAR*D Child Study}

Table 5 shows the demographic and clinical characteristics of the treatment-seeking mothers and their children in the current study (Ottawa and New York City) compared to the STAR ${ }^{\star} \mathrm{D}$ Child study conducted in seven U.S. sites (Boston, San Diego, Ann Arbor, Chapel Hill, Dallas, Nashville, and Richmond; details of STAR ${ }^{*} \mathrm{D}$ Child methods are provided in Miranda et al. ${ }^{10}$ ). The diagnostic assessments for the children were identical and were under the supervision of the same team in New York for both studies. Mothers in the $\mathrm{STAR}^{\star} \mathrm{D}$ Child study were significantly younger (37 versus 41 years of age), had higher education levels, a lower proportion of Hispanic and higher proportion of black participants; were more often employed full-time, had higher incomes, and had higher baseline HAMD-17 scores but no significant difference in proportion with severe depression. The children in both studies were the same mean age (11 years), had the same proportion living in two-parent households $(53 \%)$, and had the same proportion of female participants (48\%).

Despite the differences in demographics between studies and different sites, there were no statistically significant differences in prevalence of current or lifetime psychiatric disorders in the children between these studies (Table 6). The current prevalence rates of any offspring psychiatric disorder in STAR ${ }^{*} \mathrm{D}$-Child and the current study were $34 \%$ and $42 \%$, respectively, and lifetime prevalence rates were $45 \%$ and $47 \%$. There were no significant differences between children in overall functioning (C-GAS).

\section{DISCUSSION}

We examined the current psychopathology of children aged 7 through 17 years of currently 
TABLE 5 Comparison of Demographic Characteristics of Mothers and Children in Sequenced Treatment Alternatives to Reduce Depression (STAR*D-Child) vs. the Current Study ${ }^{a}$

\begin{tabular}{|c|c|c|c|}
\hline Mothers & $\begin{array}{c}\text { STAR*D-Child Study } \\
(\mathbf{n}=151)\end{array}$ & $\begin{array}{l}\text { Current Study } \\
\quad(n=82)\end{array}$ & Statistic $^{\mathrm{b}}$ \\
\hline Age, mean (SD) & $37.1(6.6)$ & $41(6.3)$ & $-4.38^{* *}$ \\
\hline \multicolumn{4}{|l|}{ Education, n (\%) } \\
\hline Some high school or less & 21 (13.9) & $17(20.7)$ & \multirow[t]{4}{*}{$11.46^{* *}$} \\
\hline High school graduate & 29 (19.2) & $19(23.2)$ & \\
\hline Some college & 69 (45.7) & $19(23.2)$ & \\
\hline College graduate & $32(21.2)$ & $26(31.7)$ & \\
\hline \multicolumn{4}{|l|}{ Ethnicity, n (\%) } \\
\hline White & 67 (44.4) & $38(46.3)$ & \multirow[t]{4}{*}{$47.78^{* *}$} \\
\hline Black & $56(37.1)$ & $2(2.4)$ & \\
\hline Hispanic & $22(14.6)$ & $39(47.6)$ & \\
\hline Other & $6(4)$ & $3(3.7)$ & \\
\hline \multicolumn{4}{|l|}{ Marital status, $\mathrm{n}(\%)$} \\
\hline Married with spouse & $64(42.4)$ & $34(41.5)$ & \multirow[t]{2}{*}{0.00} \\
\hline Single/divorced/separated & 87 (57.6) & 47 (57.3) & \\
\hline \multicolumn{4}{|l|}{ Employment status, n (\%) } \\
\hline Employed full-time/part-time & 98 (64.9) & 45 (54.9) & \multirow[t]{4}{*}{$20.93^{* *}$} \\
\hline Homemaker & 20 (13.2) & $4(4.9)$ & \\
\hline Fulltime student/unemployed & $31(20.5)$ & $24(29.3)$ & \\
\hline Other & $0(0)$ & $8(9.8)$ & \\
\hline Receiving public assistance, $n(\%)$ & $43(28.5)$ & $30(36.6)$ & 1.62 \\
\hline \multicolumn{4}{|l|}{ Household income, n (\%) } \\
\hline Less than $\$ 15 \mathrm{~K}$ & $38(25.2)$ & $29(35.4)$ & \multirow[t]{3}{*}{$14.27^{\star *}$} \\
\hline$\$ 15-39 K$ & $58(38.4)$ & $12(14.6)$ & \\
\hline More than $\$ 40 \mathrm{~K}$ & $49(32.5)$ & $37(45.1)$ & \\
\hline \multicolumn{4}{|l|}{ Clinical characteristics } \\
\hline HAMD-17, mean (SD) & $24.7(5.0)$ & $21.9(4.4)$ & $4.25^{* *}$ \\
\hline \multicolumn{4}{|l|}{ MDD severity (current), $\mathrm{n}(\%)^{\mathrm{c}}$} \\
\hline Moderate & $42(27.8)$ & 27 (32.9) & \multirow[t]{2}{*}{1.27} \\
\hline Severe & $109(72.2)$ & $50(61)$ & \\
\hline Children & $(n=151)$ & $(n=145)$ & Statistic $^{b}$ \\
\hline Age, mean $(S D)$ & $11.5(2.8)$ & $11.9(3.3)$ & -1.13 \\
\hline \multicolumn{4}{|l|}{ Living status, n (\%) } \\
\hline Two-parent household & 79 (52.3) & $78(53.8)$ & \multirow[t]{2}{*}{0.06} \\
\hline Mother only & 72 (47.7) & $67(46.2)$ & \\
\hline Female, n (\%) & $72(47.7)$ & $70(48.3)$ & 1.45 \\
\hline \multicolumn{4}{|l|}{ Education, n (\%) } \\
\hline Grade 1-6 & $81(53.6)$ & $67(46.2)$ & \multirow[t]{2}{*}{1.96} \\
\hline Above 6th Grade & $68(45)$ & $78(53.8)$ & \\
\hline \multicolumn{4}{|c|}{ 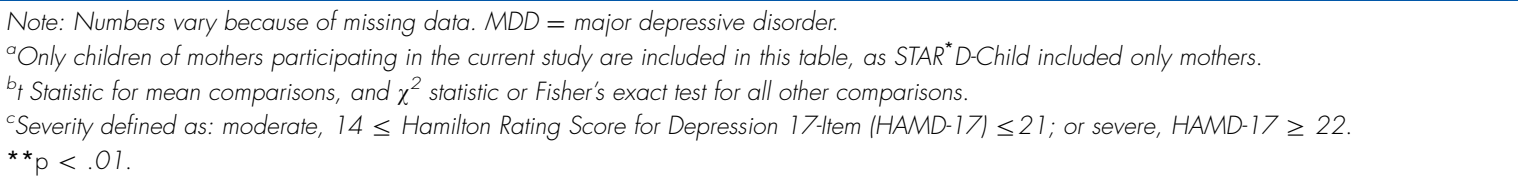 } \\
\hline
\end{tabular}

depressed mothers seeking treatment in two sites, New York City and Ottawa. Many of these children were highly symptomatic and had impaired functioning. Greater than one-third of the children of depressed mothers in both sites were diagnosed with at least one current psychiatric illness. Severity of mothers' depression predicted higher rates of behavioral disorders in their children. Maternal comorbid anxiety disorder predicted higher rates of children's anxiety 
TABLE 6 Children's Current and Lifetime Diagnoses in the Current Study and in Sequenced Treatment Alternatives to Reduce Depression (STAR*D-Child) ${ }^{a}$, Among Children of Depressed Mothers

\begin{tabular}{|c|c|c|c|c|c|c|}
\hline & \multicolumn{3}{|c|}{ Current Diagnosis } & \multicolumn{3}{|c|}{ Lifetime Diagnosis } \\
\hline & n (\%) & $\begin{array}{c}\text { STAR* } \\
\text { D-Child } \\
\text { n (\%) }\end{array}$ & $\begin{array}{l}\text { Current Study } \\
\text { Statistic }^{b}\end{array}$ & $\begin{array}{c}\text { STAR* }^{*} \\
\text { D-Child } \\
\text { n (\%) }\end{array}$ & $\begin{array}{c}\text { Current } \\
\text { Study } \\
\text { n (\%) }\end{array}$ & Statistic $^{b}$ \\
\hline Affective disorder, any & $15(9.9)$ & $22(15.2)$ & 1.86 & $28(18.5)$ & $31(21.4)$ & 0.37 \\
\hline Major depressive disorder & $8(5.3)$ & $13(9)$ & 1.51 & $15(9.9)$ & $21(14.5)$ & 1.43 \\
\hline Other depressive disorders ${ }^{c}$ & $7(4.6)$ & $7(4.8)$ & 0.01 & $14(9.3)$ & $8(5.5)$ & 1.52 \\
\hline Anxiety disorder, any & $24(15.9)$ & 28 (19.3) & 0.60 & 30 (19.9) & $35(24.1)$ & 0.79 \\
\hline Separation anxiety disorder & $7(4.6)$ & $14(9.7)$ & 2.83 & $13(8.6)$ & 15 (10.3) & 0.26 \\
\hline Social phobia & $8(5.3)$ & $5(3.4)$ & 0.60 & $8(5.3)$ & $6(4.1)$ & 0.22 \\
\hline Other anxiety disorders ${ }^{d}$ & 5 (3.3) & $8(5.5)$ & 0.86 & $7(4.6)$ & 10 (6.9) & 0.70 \\
\hline Behavioral disorder, any ${ }^{\mathrm{e}}$ & 33 (21.9) & $34(23.4)$ & 0.11 & $43(28.5)$ & $39(26.9)$ & 0.09 \\
\hline ADHD & $26(17.2)$ & $29(20)$ & 0.38 & 33 (21.9) & $31(21.4)$ & 0.01 \\
\hline $\begin{array}{l}\text { Oppositional defiant } \\
\text { disorder }\end{array}$ & $10(6.6)$ & $3(2.1)$ & 3.65 & $15(9.9)$ & $15(10.3)$ & 0.01 \\
\hline Conduct disorder & $2(1.3)$ & $3(2.1)$ & 0.25 & $3(2)$ & $6(4.1)$ & 1.16 \\
\hline Disorder, any & $52(34.4)$ & $61(42.1)$ & 1.83 & $68(45)$ & $68(46.9)$ & 0.10 \\
\hline $\begin{array}{l}\text { Global functioning at baseline, } \\
\text { mean }(S D)^{f}\end{array}$ & $70(11.7)$ & $72.7(13.8)$ & -1.80 & - & - & - \\
\hline $\begin{array}{l}\text { Note: Numbers may vary because of m } \\
\text { Behavior Checklist. } \\
{ }^{a} \text { A prior study of children of depressed } \\
b_{\text {bt statistic for mean comparisons, and }} \\
{ }^{c} \text { Includes depressive disorder not otherv } \\
\text { depressed mood. } \\
\text { dIncludes generalized anxiety disorder, } \\
\text { eIncludes attention-deficit/hyperactivity } \\
{ }^{f} \text { Based on the Child-Global Assessment }\end{array}$ & $\begin{array}{l}\text { g data. There w } \\
\text { ners referenced } \\
\text { tistic or Fisher's } \\
\text { specified (NOS, } \\
\text { assive-compulsiv } \\
\text { der (ADHD), op }\end{array}$ & $\begin{array}{l}\text { e no significant di } \\
\text { the text. } \\
\text { xact test for all oth } \\
\text { adjustment disorde } \\
\text { disorder, and anx } \\
\text { ositional disorder, }\end{array}$ & $\begin{array}{l}\text { ences between stud } \\
\text { omparisons. } \\
\text { ith depressed moo } \\
\text { disorder NOS. } \\
\text { conduct disorder. }\end{array}$ & Ind adjustment & order with mixe & anxiety and \\
\hline
\end{tabular}

disorders and any disorder. These high rates of psychiatric disorders in the children of treatmentseeking depressed mothers are consistent with studies of offspring at high risk due to parents' depression $^{1,2}$ as well as studies of depressed mothers coming to primary care. ${ }^{19}$

The prevalence rates of the children's disorders when the depressed mother came for treatment in the midst of a depressive episode were consistent with our previous study (STAR ${ }^{\star}$ D Child), which used similar diagnostic methods for the children. ${ }^{10}$ Despite the considerable site and maternal demographic differences, the prevalence of current and lifetime psychiatric disorders in the children were similarly elevated in both studies and across sites, suggesting that the relationship may hold across demographics and culture. Both studies found that maternal comorbid anxiety disorders were associated with an increased risk of children's psychiatric disorders. Variability in the prediction of childhood disorders based on maternal depression subtypes (e.g., melancholic depression predicted childhood anxiety disorders in the current study but not in STAR ${ }^{*} \mathrm{D}$ ) is likely due to maternal clinical variations and subtype definitions between studies. The current study used the SCID interview with the mother to detect depressive subtypes and STAR ${ }^{\star} \mathrm{D}$ used items from the HAMD-17. Data from these studies suggest that children of depressed mothers are at high risk for current and lifetime psychiatric disorders when mothers come for treatment. The finding that maternal comorbid anxiety disorders were associated with higher rates of anxiety disorders and other disorders in the children is consistent with studies showing the adverse effect of comorbid anxiety and MDD on clinical course and treatment outcome. ${ }^{20,21}$

Despite considerable efforts, we had difficulty recruiting depressed parents in both studies. In STAR ${ }^{\star} \mathrm{D}$ Child at seven U.S. sites, $22 \%$ of the recruited women who were aged 25 through 
60 years had children or adolescents aged 7 through 17 years. In our current study of depressed mothers, about $34 \%$ had children or adolescents aged 7 through 17 years. This figure is slightly overstated, as in the last 2 months of the study we selectively recruited parents with children, resulting in two additional mothers. The low proportion of parents of children in this age group in this and our previous study (STAR ${ }^{\star} \mathrm{D}$-Child) might be the consequence of having a significant proportion of low-income mothers in both studies. Some reports suggest that low-income parents do not use treatment services for themselves even if these services are available to them, and require outreach for childcare, transportation, and flexible schedules. 22,23

Study limitations should be noted. Our findings of current prevalence apply to children of treatment-seeking mothers with moderate to severe MDD, and may not apply to more mildly ill mothers or to community samples. We did not examine the prevalence of all child psychiatric disorders, and included only 18 DSM-IV childhood disorders. The choice of disorders examined in the children was guided by the research on children of depressed mothers. The data are crosssectional and cannot be seen as reflective of causal pathways. A control group of children of mothers who were not depressed and were coming for treatment for a nonpsychiatric disorder would have strengthened the comparison. Potential reporting bias of depressed mothers is a serious concern. We took several precautions such as having separate mother/child interviews, and interviewing children before the mother's interview. We also examined the relationship between the mean number of child-reported symptoms (based on K-SADS) and the mother's severity of maternal depression based on the HAMD-17. We found a strong relationship between childreported symptoms and severity of maternal depression in both sites. This suggests that the association between severity of maternal depression and the number of child K-SADS-PL symptoms (more symptoms among children of severely as compared to moderately depressed mothers) is not merely an artifact of reporting by depressed mothers.

The clinical implications of these findings are that clinicians treating depressed mothers should inquire about child psychiatric symptoms and functioning, especially if the mother has an anxiety disorder or is severely depressed. Clinicians of children with psychiatric symptoms should also inquire about psychiatric symptoms in their parents. ${ }^{24}$ Asking depressed mothers about their children may be particularly useful, as an increasing number of studies have shown the close relationship between successful treatment of the mother's depression, whether by medication or psychotherapy, and relief of the child's symptoms. ${ }^{5-8,25-29}$ Although the evidence is based mostly on studies of children of depressed mothers, these findings might also be applicable to children of depressed fathers. The finding that comorbid anxiety disorders in the mothers predicted higher rates of disorders in her children is important, as there is considerable evidence that comorbid anxiety disorder in a depressed adult contributes to poorer antidepressant response. ${ }^{30}$ Thus, mothers who are both depressed and anxious, and their children, require extra attention. $\varepsilon$

\section{CG Clinical Guidance}

- Children and adolescents aged 7 to 17 years of mothers coming for the treatment of depression are at high risk for having psychiatric symptoms and behavioral problems.

- Clinicians treating depressed mothers should inquire about the children's emotional state and about behavioral problems.

- Monitoring of the child's symptoms during the course of the mother's treatment is warranted.

- Referral for treatment of the child should be considered if the child's symptoms are not alleviated after a remission of maternal depression.

- Comorbid maternal anxiety disorders or severe maternal depression may be associated with increased problems in the children.

Accepted August 23, 2012

Dr. Batten and Blier is with the University of Ottawa Institute of Mental Health Research. Dr. Batten is with the Carleton University, Ottaway. Drs. Hernandez, Pilowsky, Stewart, Poh, Wickramaratne, and Weissman are with the New York State Psychiatric Institute. Drs. Pilowsky, Stewart, Wickramaratne, and Weissman are with the College of Physicians and Surgeons at Columbia University. Drs. Pilowsky, Wickramaratne, and Weissman are with the Mailman School of Public Health at Columbia University. Dr. Flament is with the Youth Research Unit, University of OHawa

Clinical guidance is available at the end of this article.

This study was funded by National Institute of Mental Health (NIMH) grants MH082255 (M.M.W., PI), MH076961-01A2 U.W.S., PI), and MH077285-01A2 (P.B., PI); and by a Canadian Institutes of Health Research Ph.D. Scholarship (L.A.B.).

Dr. Wickramaratne served as the statistical expert for this research. 
The authors thank Delphie Dugal-Tessier at the University of Ottawa for conducting clinical interviews, and Dr. Richard Bergeron and his team at Pierre-Janet Hospital in Gatineau, Québec, for their participation. Disclosure: Dr. Pilowsky has received a speaker's fee from AstraZeneca in connection with an oral presentation at the Annual Meeting of the Psychiatric Association of Quebec, Canada, and has received grant support from NIMH and the National Institute on Drug Abuse (NIDA). Dr. Stewart has received grant support from NIMH, supply of study medications from Forest Laboratories, and honoraria from Forest Laboratories, Pfizer, Alkermes, and Shire. Dr. Blier has received research funding from Lundbeck, AstraZeneca, Janssen, Servier, and Bristol-Myers Squibb. He has been involved in clinical studies supported by Bristol-Myers Squibb and Roche. He has served as a consultant for Otsuka, Pfizer, Eli Lilly and Co., and Lundbeck. He has received honoraria from Servier and from Lundbeck. Dr. Weissman has received funding from NIMH, NIDA, the National Alliance for Research on Schizophrenia and Depression (NARSAD), the Sackler

\section{REFERENCES}

1. Beardslee WR, Gladstone TR, O'Connor EE. Transmission and prevention of mood disorders among children of affectively ill parents: a review. J Am Acad Child Adolesc Psychiatry. 2011; 50:1098-1109.

2. Weissman MM, Wickramaratne P, Nomura Y, et al. Families at high and low risk for depression: a 3-generation study. Arch Gen Psychiatry. 2005;62:29-36.

3. Wickramaratne PJ, Weissman MM. Onset of psychopathology in offspring by developmental phase and parental depression. J Am Acad Child Adolesc Psychiatry. 1998;37:933-942.

4. Wang PS, Lane M, Olfson M, Pincus HA, Wells KB, Kessler RC Twelve-month use of mental health services in the United States: results from the National Comorbidity Survey Replication. Arch Gen Psychiatry. 2005;62:629-640.

5. Gunlicks ML, Weissman MM. Change in child psychopathology with improvement in parental depression: a systematic review. J Am Acad Child Adolesc Psychiatry. 2008;47:379-389.

6. Pilowsky DJ, Wickramaratne P, Talati A, et al. Children of depressed mothers 1 year after the initiation of maternal treatment: findings from the STAR ${ }^{\star} \mathrm{D}$-Child Study. Am J Psychiatry. 2008; $165: 1136-1147$

7. Weissman MM, Pilowsky DJ, Wickramaratne PJ, et al. Remissions in maternal depression and child psychopathology: a STAR ${ }^{\star} \mathrm{D}$ child report. JAMA. 2006;295:1389-1398.

8. Wickramaratne P, Gameroff MJ, Pilowsky DJ, et al. Children of depressed mothers 1 year after remission of maternal depression: findings from the STAR ${ }^{\star} \mathrm{D}-$ Child study. Am J Psychiatry. 2011; 168:593-602.

9. Talati A, Wickramaratne PJ, Pilowsky DJ, et al. Remission of maternal depression and child symptoms among single mothers: a STAR ${ }^{\star}$ D-Child report. Soc Psychiatry Psychiatr Epidemiol. 2007; 42:962-971.

10. Pilowsky DJ, Wickramaratne PJ, Rush AJ, et al. Children of currently depressed mothers: a STAR ${ }^{\star} \mathrm{D}$ ancillary study. J Clin Psychiatry. 2006;67:126-136.

11. Rush AJ, Fava M, Wisniewski SR, et al. Sequenced treatment alternatives to relieve depression $\left(\mathrm{STAR}^{\star} \mathrm{D}\right)$ : rationale and design. Control Clin Trials. 2004:25:119-142.

12. First MB, Gibbon M, Williams JBW. Structured Clinical Interview for DSM-IV Axis I Disorders (SCID-I). In: Rush AJ, First MB, Blacker D, eds. Handbook of Psychiatric Measures. Washington DC: American Psychiatric Press; 2008: p. 40-43.

13. Davidson J, Turnbull CD, Strickland R, Miller R, Graves K. The Montgomery-Asberg Depression Scale: reliability and validity. Acta Psychiatr Scand. 1986;73:544-548.

14. Hamilton M. Development of a rating scale for primary depressive illness. Br J Soc Clin Psychol. 1967;6:278-296.

15. Kaufman J, Birmaher B, Brent D, et al. Schedule for Affective Disorders and Schizophrenia for School-Age Children-Present and
Foundation, and the Interstitial Cystitis Association, and has received royalties from Oxford University Press, Perseus Press, American Psychiatric Association Press, and Multihealth Systems. Ms. Batten, Ms. Hernandez, and Mr. Poh, and Drs. Flament and Wickramaratne report no biomedical financial interests or potential conflicts of interest.

Correspondence to Myrna M. Weissman, Ph.D., Professor of Epidemiology in Psychiatry, College of Physicians and Surgeons, Columbia University, Mailman School of Public Health, Chief, Division of Epidemiology, New York State Psychiatric Institute, Unit 24, 1051 Riverside Drive, New York, NY 10032; e-mail: mmw3@columbia.edu

0890-8567/\$36.00/@ 2012 American Academy of Child and Adolescent Psychiatry http://dx.doi.org/10.1016/i.jaac.2012.08.020

Lifetime Version (K-SADS-PL): initial reliability and validity data. J Am Acad Child Adolesc Psychiatry. 1997;36:980-988.

16. Shaffer D, Gould MS, Brasic J, et al. A children's global assessment scale (CGAS). Arch Gen Psychiatry. 1983;40:1228-1231.

17. Liang KY, Zeger SL. Longitudinal data anlysis using generalized linear models. Biometrika. 1986;73:13-22.

18. SAS Institute. SAS/STAT software: changes and enhancements through Release 9.3. Cary, NC: SAS Institute; 2012.

19. Weissman MM, Feder A, Pilowsky DJ, et al. Depressed mothers coming to primary care: maternal reports of problems with their children. J Affect Disord. 2004;78:93-100.

20. Brown C, Schulberg HC, Madonia MJ, Shear MK, Houck PR. Treatment outcomes for primary care patients with major depression and lifetime anxiety disorders. Am J Psychiatry. 1996; 153:1293-1300.

21. Feske U, Frank E, Kupfer DJ, Shear MK, Weaver E. Anxiety as a predictor of response to interpersonal psychotherapy for recurrent major depression: an exploratory investigation. Depress Anxiety. 1998;8:135-141.

22. Miranda J, Siddique J, Belin TR, Kohn-Wood LP. Depression prevalence in disadvantaged young black women-African and Caribbean immigrants compared to US-born African Americans. Soc Psychiatry Psychiatr Epidemiol. 2005;40:253-258.

23. Verdeli H, Ferro T, Wickramaratne P, Greenwald S, Blanco C, Weissman MM. Treatment of depressed mothers of depressed children: pilot study of feasibility. Depress Anxiety. 2004;19:51-58.

24. Kennard BD, Hughes JL, Stewart SM, et al. Maternal depressive symptoms in pediatric major depressive disorder: relationship to acute treatment outcome. J Am Acad Child Adolesc Psychiatry. 2008;47:694-699.

25. Birmaher B. Remission of a mother's depression is associated with her child's mental health. Am J Psychiatry. 2011;168:563-565.

26. Coiro MJ, Riley A, Broitman M, Miranda J. Effects on children of treating their mothers' Depression: results of a 12-month followup. Psychiatr Serv. 2012;63:357-363.

27. Garber J, Ciesla JA, McCauley E, Diamond G, Schloredt KA. Remission of depression in parents: links to healthy functioning in their children. Child Dev. 2011;82:226-243.

28. Siegenthaler E, Munder T, Egger M. Effect of preventive interventions in mentally ill parents on the mental health of the offspring: systematic review and meta-analysis. J Am Acad Child Adolesc Psychiatry. 2012;51(8-17):e18.

29. Swartz HA, Frank E, Zuckoff A, et al. Brief interpersonal psychotherapy for depressed mothers whose children are receiving psychiatric treatment. Am J Psychiatry. 2008;165:1155-1162.

30. Carter GC, Cantrell RA, Victoria Z, et al. Comprehensive review of factors implicated in the heterogeneity of response in depression. Depression and anxiety. 2012;29:340-354. 